\title{
DERIVAÇÃO DE ESCALAS DE TEMPO LAGRANGEANAS DEPENDENTES DA DISTÂNCIA DA FONTE NA CAMADA LIMITE PLANETÁRIA NEUTRA
}

\author{
Franco C. Degrazia ${ }^{1}$, Gervásio A. Degrazia ${ }^{2}$, Marco Tullio M. B. de Vilhena ${ }^{1}$ \\ ${ }^{1}$ UFRGS/PROMEC, Porto Alegre, RS \\ ${ }^{2}$ Departamento de Física/UFSM, Santa Maria, RS
}

\section{INTRODUÇÃO}

O objetivo deste trabalho é propor uma nova formulação para o tempo de descorrelação Lagrangeano em função da distância da fonte para uma turbulência não homogênea na camada limite planetária neutra (CLPN). As escalas de tempo de descorrelação são escalas características dos vórtices que possuem a energia principal no processo de difusão turbulenta. As escalas de tempo de descorrelação podem variar no espaço e com o tempo de viagem do contaminante. Próximo à fonte, as partículas de fluido retém a memória do ambiente turbulento local. Conhecer esse efeito é fundamental para avaliar a dispersão de contaminantes, principalmente próximo à fonte, onde são observadas as maiores concentrações de contaminantes. Em modelos Lagrangeanos, as variáveis fundamentais são a variância ae velocidade do vento e as escalas de tempo de descorrelação (Anfossi e Physick, 2005).

\section{DERIVAÇÃO DE ESCALAS}

O presente modelo emprega a seguinte equação para a evolução da escala de tempo de descorrelação Lagrangeano $T_{L i}$ Batchelor (1949)

$$
T_{L i}=\frac{\beta_{i}}{2 \pi} \int_{0}^{\infty} F_{i}^{E}(n) \frac{\operatorname{sen}\left(2 \pi n t / \beta_{i}\right)}{n} d n
$$

com $i=u, v \mathrm{e}^{w}$ sendo as componentes ortogonais, onde $F_{i}^{E}(n)$ é espectro Euleriano de energia normalizado pela variância da velocidade do vento $\sigma_{i}^{2}, \beta_{i}$ é o fator de escala definido como a razão entre as escalas de tempo integral Lagrangeana e Euleriana, $n$ é a frequência, e $t$ é o tempo de viagem. Do ponto de vista físico, $F_{i}{ }^{(}(n)$ descreve a energia cinética contida no escoamento distribuída entre as distintas frequências. $\mathrm{O}$ espectro de velocidade Euleriano sob condições atmosféricas neutras pode ser descrito como (Degrazia e Anfossi, 1998):

$$
\frac{n S_{i}(n)}{\mu_{*}^{2}}=\frac{1.5 C_{i} \Phi_{\varepsilon}^{2 / 3} f}{\left[1+\frac{1.5 f^{5 / 3}}{\left(f_{m}\right)_{i}^{5 / 3}}\right]\left(f_{m}\right)_{i}^{5 / 3}}
$$

onde $C_{i}=\alpha_{i}(0.50 \pm 0.02)(2 \pi \kappa)^{-2 / 3} ; \quad \alpha_{i}=1 / 4,4 / 3$ e $4 / 3$ para as componentes $u, v$ e $w$, respectivamente; $\kappa=0.4$ é a constante de von Karman, $f=n z / U$ é a frequência adimensional ( $n$ sendo a frequência cíclica, $U$ a velocidade horizontal média do vento e $z$ é a altura da observação), $\left(f_{m}\right)_{i}$ é a frequência adimensional de pico espectral neutro e $\mu_{*}^{2}=\left(\mu_{* 0}\right)^{2}[1-(z / h)]^{1.7}$ é a velocidade 
de fricção local para uma CLP neutra com ${ }^{\mu_{*} 0}$ sendo a velocidade de fricção superficial e ${ }^{h}$ é a altura da CLPN. A taxa de dissipação adimensional é definida como $\Phi_{\varepsilon}=k z \varepsilon /\left(u_{*}\right)_{0}^{3}$.

A integração analítica da equação (2) sobre todo o domínio da frequência estabelece a variância da velocidade Euleriana turbulenta e foi determinada como (Degrazia e Anfossi.1998):

$\sigma_{i}^{2}=\frac{2.32 C_{i} \Phi_{\varepsilon}^{2 / 3} u_{*}^{2}}{\left(f_{m}\right)_{i}^{2 / 3}}$

a qual é utilizada para normalizar o espectro, portanto o espectro Euleriano pode ser escrito como (Degrazia e Anfossi.1998):

$F_{i}^{E}=\frac{S_{i}(n)}{\sigma_{i}^{2}}=\frac{0.64 \frac{z}{U}}{\left(f_{m}\right)_{i}}\left\{1+1.5\left(\frac{n z}{U\left(f_{m}\right)_{i}}\right)^{5 / 3}\right\}^{-1}$.

Substituindo as equações (3) e (4) em (1) e considerando $\beta_{i}=0.55 U / \sigma_{i}$, se estabelece a

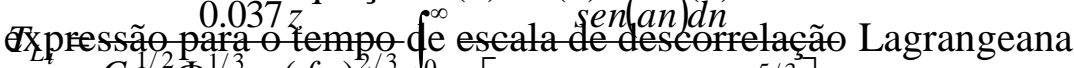
$C_{i}^{1 / 2} \Phi_{\varepsilon}^{1 / 3} u_{*}\left(f_{m}\right)_{i}^{2 / 3}$

$$
n\left[1+1.5\left(\frac{n z}{U\left(f_{m}\right)_{i}}\right)^{5 / 3}\right]
$$

Os demais termos da equação (1) são definidos como

$$
\begin{aligned}
& \frac{\beta_{i}}{2 \pi}=\frac{0.057 U\left(f_{m}\right)_{i}^{1 / 3}}{C_{i}^{1 / 2} \Phi_{\varepsilon}^{1 / 3} u_{*}} \\
& \frac{2 \pi t}{\beta_{i}}=a=\frac{17.4 U C_{i}^{1 / 2} \Phi_{\varepsilon}^{1 / 3}\left(f_{m}\right)_{i}^{1 / 3}}{\left(f_{m}\right)_{i}^{1 / 3}} \frac{z}{U} \frac{X u_{*}}{U z},
\end{aligned}
$$

onde uma transposição do tempo para o espaço é aplicada na dependência temporal da equação (5), sendo o tempo de viagem definido por $X / U$ (Alves et al., 2012).

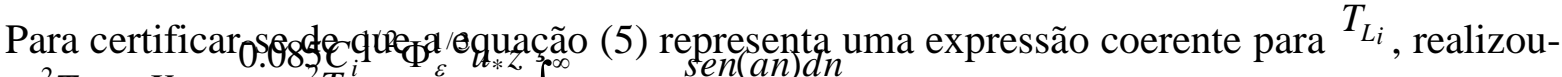

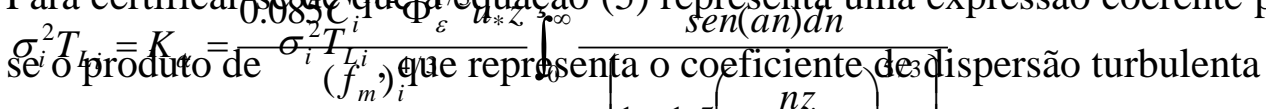

$$
n\left\{1+1.5\left(\frac{n z}{U\left(f_{m}\right)_{i}}\right)\right\}
$$

A equação (6) está de acordo com expressão de $K_{\alpha}$ presente em Alves et al., (2012). 


\section{CONCLUSÃO}

$\mathrm{Na}$ continuação deste trabalho, uma solução numérica para $T_{L i}$, eq. (5) será empregada em conjunto com o modelo de dispersão estocástico Lagrangeano LAMBDA (Ferrero e Anfossi, 1998) para simular o campo de concentração de contaminantes. Estas escalas de tempo de descorrelação serão validadas com dados de concentração medidos em experimentos no campo próximo, intermediário e distante de uma fonte pontual contínua.

\section{REFERÊNCIAS BIBLIOGRÁFICAS}

ALVES, I. P.; DEGRAZIA, G. A.; BUSKE, D.; VILHENA, M. T.; MORAES, O. L. L.; ACEVEDO, O. C. Derivation of an Eddy Diffusivity Coefficient Depending on a Source Distance for Shear Dominated Planetary Boundary Layer, Physica A: Statistical Mechanics and its Applications, v. 391, p. 6577-6586, 2012.

ANFOSSI, D., PHYSICK, W. Lagrangian Particle Models, In: ZANNETTI, Paolo. Air Quality Modeling, Advanced Topics. The EnviroComp Institute, Air and Waste Management Association, 2005, v. 2, cap. 11.

BATCHELOR, G. K., Diffusion in a Field of Homogeneous Turbulence I. Eulerian analysis, Aust. J. Sci. Res. Series A, v.2, p. 437-450, Phys. Sci., 1949.

FERRERO E.; ANFOSSI D. Comparison of PDFs, Closures Schemes and Turbulence Parameterizations in Lagrangian Stochastic Models. Int. J. Environment and Pollution, v. 9, p. 384-410, 1998.

DEGRAZIA, G.A., ANFOSSI, D. Estimation of the kolmogorov Constant C0 from Calssical statistical diffusion theory. Atmospheric Environment, v.32, 3611-3614 